\title{
Simulation for DFB Lasers with Grating Phase of $\pi$ on One Mirror Face
}

\author{
Kee-Young Kwon'1)
}

\begin{abstract}
In this paper, a simulation tool has been developed and the threshold gain and lasing frequency of a lasing mode in longitudinal direction of a $1.55 \mathrm{um}$ DFB(Distributed Feedback) laser with two mirrors and without anti-reflection coatings, that have both gain and index gratings, have been analyzed. The main purpose of this paper is to find an optimum value of grating phase of a mirror face and coupling strength in order to obtain excellent stable frequency operation of a single-mode during high-speed modulation. The grating phase of a left mirror face is fixed to be $\pi$ and the grating phase of a right mirror face is varied. In order to obtain the largest gap in the oscillation threshold-gain between the first and second modes, DFB lasers should have coupling strength $\kappa L=10$ in case of Bragg deviation $\delta L>0$. In case of $\delta L<0$, the oscillation frequency selectivity of the single-mode is excellent in the range from $\kappa L=2$ to $\kappa L=6$, except of $\rho_{r}$ phase $=0$. If the goal is to minimize the oscillation gain, DFB lasers should have $\kappa L=10$, in order to enhance the backward scattering of the propagating light.
\end{abstract}

Keywords: DFB Lasers, Oscillation Gain, Grating Phase, Index Grating, Gain Grating

\section{Introduction}

Light sources for wideband optical communication systems should be excellent in frequency selectivity and stable in frequency characteristics even at high-speed modulation[1]. In particular, as laser light sources used in high-speed broadband optical communication systems, DFB lasers have a single-mode operation, low frequency change, and stable frequency operation during high frequency current modulation[2-5]. The DFB laser uses Bragg scattering to feed the light back for the oscillation, instead of the photon reflection on both mirror faces. In the case of the refractive-index coupled DFB lasers, the refractive-index grating has a finite length so the reflection on the mirror faces cannot be ignored. Therefore, in the refractive-index coupled DFB lasers, the oscillation characteristics are sensitive depending on the degenerate characteristics of the lasing wavelength and the reflectance and phase of the mirror surface. To solve this

Received(September 20, 2019), Review Result(1st: October 16, 2019, 2nd: November 30, 2019), Accepted(January 30, 2020)

1) (Professor) 31080 Div. of Electrical, Electronic and Control Engineering, Kongju National Univ., 1223-24 Cheonan-daero, Seobuk-gu, Cheonan, Chungnam, Korea

email: kky@kongju.ac.kr 
problem, a DFB laser with anti-reflective coating and phase-shifted by a quarter of a wavelength has been proposed to prevent reflection[6]. But the residual reflectance must be made smaller than 0.005 , and the problem is that spatial hole burning is so severe that the non-linearity of gain is caused[7-10].

In this paper, DFB lasers with a wavelength of $1.55 \mu \mathrm{m}$ have both index and gain gratings, simultaneously with two mirror faces and without an anti-reflective coating. While the grating phase of the left mirror face is fixed to be $\pi$, the oscillation frequency and threshold gain characteristics of DFB lasers are theoretically analyzed as the grating phase of the right mirror face is varied. The purpose of this paper is to find the optimum coupling strength $\kappa L$ values and grating phases of the right mirror face, in order to obtain single-mode operation, low frequency change, and stable frequency operation during high frequency current modulation. This issue is very important characteristics in DFB laser studies. In section 2, the basic theory is explained. The coupled-mode theory is basically used for laser model. In section 3, the oscillation frequency characteristics of DFB lasers and the gain characteristics of the oscillation modes are examined as the grating phase of the right mirror face is changed. A brief conclusion is given in section 4 .

\section{Derivation of The Equation}

\subsection{Threshold Gain and Lasing Frequency [11]}

DFB lasers have both gain and index gratings. By considering only first-order component in Fourier series, the longitudinal profiles of gain $\alpha(z)$ and refractive-index $n(z)$ can approximately be expressed as follows;

$$
\begin{aligned}
& \alpha(z)=\alpha+(\Delta \alpha) \cos \left(\frac{2 \pi z}{\Lambda}+\Omega\right) \\
& n(z)=n+(\Delta n) \cos \left(\frac{2 \pi z}{\Lambda}+\Omega\right)
\end{aligned}
$$

where $\alpha$ and $n$ are averaged gain and refractive-index, and $\Delta \alpha$ and $\Delta n$ are the amplitude variations with spatial period $\Lambda$ and phase $\Omega$. The scalar wave equation which should be satisfied in DFB lasers is given as

$$
\nabla^{2} E(z, t)+k^{2}(z) E(z, t)=0
$$


In general, the form of $E(z, t)=E(z) e^{j \omega t}$ is the solution of equation (3) and the propagating constant $k$ can be written as

$$
k^{2}(z) \approx \beta^{2}+j 2 \alpha \beta+4 \kappa \beta \cos \left(2 \beta_{o} z+\Omega\right)
$$

where $\beta_{o}=\frac{\pi}{\Lambda}, \beta=\frac{\omega}{c}$ and $\kappa=\left(\frac{\beta}{2}\right)\left(\frac{\Delta n}{n}\right)+j \frac{\Delta \alpha}{2} . \beta$ and $\beta_{o}$ are the mode and Bragg propagating constants, respectively.

In equation (4), $\alpha$ represents the net threshold gain for that oscillating mode. The oscillating frequency $\omega$ can be obtained from the speed of light $c$ and $\beta$. While maintaining the generality, the solution of equation (3) can be written as

$$
E(z)=R(z) e^{-j \beta_{o} z}+S(z) e^{j \beta_{o} z}
$$

where $R(z)$ is the complex amplitude profile of the right traveling wave and $S(z)$ of the left traveling wave. The following equation is obtained from equations (5) and (3).

$$
\begin{gathered}
e^{-j \beta_{o} z}\left[-j 2 \beta_{o} R^{\prime}-\beta_{o}^{2} R+\left(\beta^{2}+j 2 \alpha \beta\right) R\right]+e^{j \beta_{o} z}\left[j 2 \beta_{o} S^{\prime}-\beta_{o}^{2} S+\left(\beta^{2}+j 2 \alpha \beta\right) S\right] \\
+2 \kappa \beta\left(e^{j 2 \beta_{o} z} e^{j \Omega}+e^{-j 2 \beta_{o} z} e^{-j \Omega}\right)\left(R e^{-j \beta_{o} z}+S e^{j \beta_{o} z}\right)=0
\end{gathered}
$$

Therefore the following coupled mode equations are obtained.

$$
\begin{gathered}
-R^{\prime}+(\alpha-j \delta) R=j \kappa e^{-j \Omega} S \\
S^{\prime}+(\alpha-j \delta) S=j \kappa e^{j \Omega} R
\end{gathered}
$$

where $\delta=\beta-\beta_{o}$ gives the Bragg deviation, $|\delta| \ll \beta$ is assumed. In equations (7) and (8), $\kappa$ represents the coupling coefficient. The solution of these coupled mode equations (7) and (8) has the form

$$
\begin{aligned}
& R(z)=R_{1} e^{\gamma z}+R_{2} e^{-\gamma z} \\
& S(z)=S_{1} e^{\gamma z}+S_{2} e^{-\gamma z}
\end{aligned}
$$

where $R_{1}, R_{2}, S_{1}, S_{2}$ are constants and $\gamma$ is the complex propagating constant to be determined. Inserting (9) and (10) in (7) and (8), one obtains 


$$
\begin{aligned}
\widehat{\Gamma} R_{1} & =j \kappa e^{-j \Omega} S_{1} \\
\Gamma R_{2} & =j \kappa e^{-j \Omega} S_{2} \\
\Gamma S_{1} & =j \kappa e^{j \Omega} R_{1} \\
\widehat{\Gamma} S_{2} & =j \kappa e^{j \Omega} R_{2}
\end{aligned}
$$

$\Gamma$ and $\hat{\Gamma}$ are denoted as

$$
\begin{gathered}
\hat{\Gamma}=-\gamma+\alpha-j \delta \\
\Gamma=\gamma+\alpha-j \delta
\end{gathered}
$$

and the squared complex propagating constant $\gamma^{2}$ is given by

$$
\gamma^{2}=(\alpha-j \beta)^{2}+\kappa^{2}
$$

The boundary conditions at both the left face $z=-\frac{L^{\prime}}{2}$ and the right face $z=\frac{L^{\prime \prime}}{2}$ are written as

$$
\begin{gathered}
e^{j \beta_{o} L^{\prime} / 2}\left(R_{1} e^{-\gamma L^{\prime} / 2}+R_{2} e^{\gamma L^{\prime} / 2}\right)=\hat{\rho}_{l} e^{-j \beta_{o} L^{\prime} / 2}\left(S_{1} e^{-\gamma L^{\prime} / 2}+S_{2} e^{\gamma L^{\prime} / 2}\right) \\
e^{j \beta_{o} L^{\prime \prime} / 2}\left(S_{1} e^{\gamma L^{\prime \prime} / 2}+S_{2} e^{-\gamma L^{\prime \prime} / 2}\right)=\hat{\rho}_{r} e^{-j \beta_{o} L^{\prime \prime} / 2}\left(R_{1} e^{\gamma L^{\prime \prime} / 2}+R_{2} e^{-\gamma L^{\prime \prime} / 2}\right)
\end{gathered}
$$

where $\hat{\rho}_{l}$ and $\hat{\rho}_{r}$ are the electric field reflectivities at the left and right faces, respectively. Finally, the eigenvalue equation is written as

$$
\frac{e^{-\gamma\left(L^{\prime}+L^{\prime \prime}\right) / 2}\left(1-\rho_{l} \frac{\widehat{\Gamma}}{j \kappa}\right)}{\rho_{l}-\frac{\widehat{\Gamma}}{j \kappa}}=\frac{e^{\gamma\left(L^{\prime}+L^{\prime \prime}\right) / 2\left(\rho_{r}-\frac{\widehat{\Gamma}}{j \kappa}\right)}}{1-\rho_{r} \frac{\widehat{\Gamma}}{j \kappa}}
$$

where

$$
\begin{gathered}
\rho_{l}=\hat{\rho}_{l} e^{-j \beta_{o} L^{\prime}} e^{j \Omega} \\
\rho_{r}=\hat{\rho}_{r} e^{-j \beta_{o} L^{\prime \prime}} e^{-j \Omega}
\end{gathered}
$$

From the equations (11), (13), (15) and (16), the following formulas are derived.

$$
\gamma=\frac{-j \kappa}{2}\left(\frac{\widehat{\Gamma}}{j \kappa}-\frac{j \kappa}{\widehat{\Gamma}}\right)
$$


From the equations (20) and (23), the following eigenvalue equations can be obtained.

$$
\begin{gathered}
\gamma L=\frac{-j \kappa L \sinh (\gamma L)}{D} \times\left(\rho_{l}+\rho_{r}\right)\left(1-\rho^{2}\right) \cosh (\gamma L) \\
\pm \frac{-j \kappa L \sinh (\gamma L)}{D}\left(1+\rho^{2}\right) \times\left[\left(\rho_{l}-\rho_{r}\right)^{2} \sinh ^{2}(\gamma L)+\left(1-\rho^{2}\right)^{2}\right]^{1 / 2}
\end{gathered}
$$

where $D=\left(1+\rho^{2}\right)^{2}-4 \rho^{2} \cosh ^{2}(\gamma L), \rho^{2}=\rho_{l} \rho_{r}=\hat{\rho}_{l} \hat{\rho}_{r} e^{-j 2 \beta_{o} L}$ and $L=\left(L^{\prime}+L^{\prime \prime}\right) / 2$.

By solving (24) values of $\gamma$ can be found. These determine the propagating constants for the longitudinal lasing modes. For each $\gamma,(17)$ is used to calculate $\alpha$, which is the net threshold gain for that lasing mode, and $\delta$, which gives the oscillating frequency $\omega=\frac{c \beta}{n}=\frac{c}{n}\left(\beta_{o}+\delta\right)$ of that mode.

\section{Results and Discussions}

$3.1 \rho_{r}$ phase $=-\pi / 2$ and $\delta L>0$

[Fig. 1] shows the variation of $(\alpha L, \delta L)$ as $\kappa L$ increases from 0.1 to 10, in case of $\rho_{r}$ phase $=$ $-\pi / 2$ and $\delta L>0$. Each dot provides oscillation gain and lasing frequency information for the oscillating mode. Oscillation gain refers to the threshold gain required for that mode to oscillate.

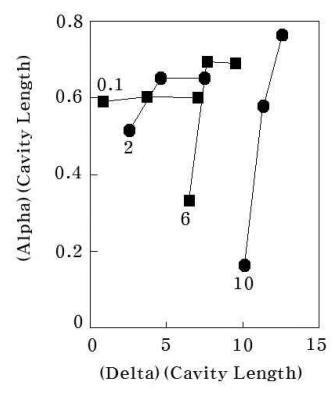

[Fig. 1] The graph of $(\alpha L, \delta L)$ in case of $\rho_{l}$ phase $=\pi, \rho_{r}$ phase $=-\pi / 2$, and $\delta L>0$ as $\kappa L$ increases

When $\kappa L$ increases from 0.1 to 10 , the threshold gain gradually decreases. It is due to the increased feedback of light. At a fixed $\kappa L$ value, the largest gap in the threshold gain between the first and second modes occurs at $\kappa L=10$. This gives single-mode operation, low frequency 
change, and stable single frequency operation during high frequency current modulation.

$3.2 \rho_{r}$ phase $=\pi$ and $\delta L>0$

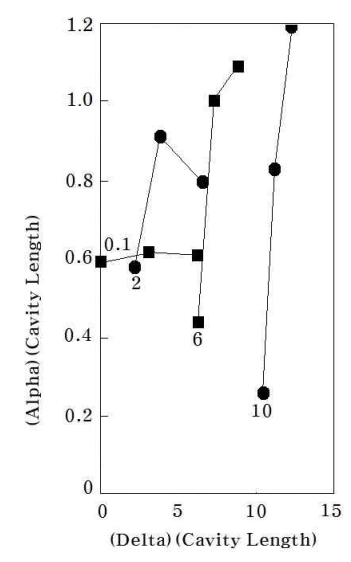

[Fig. 2] The graph of $(\alpha L, \delta L)$ in case of $\rho_{l}$ phase $=\pi, \rho_{r}$ phase $=\pi$, and $\delta L>0$ as $\kappa L$ increases

As shown in [Fig. 2], at a fixed $\kappa L$ value the largest gap in the threshold gain between the first and second modes occurs at $\kappa L=10$. At the same $\kappa L$ value, the threshold gains in case of $\rho_{r}$ phase $=\pi$ are slightly higher than those in case of $\rho_{r}$ phase $=-\pi / 2$ ([Fig. 1]). However, at a fixed $\kappa L$ value, the oscillation gain difference between the first and second modes is much higher than that in case of $\rho_{r}$ phase $=-\pi / 2$.

$3.3 \rho_{r}$ phase $=\pi / 2$ and $\delta L>0$

As shown in [Fig. 3], at a fixed $\kappa L$ value the largest gap in the oscillation gain between the first and second modes occurs at $\kappa L=10$. At the same $\kappa L$ value, the oscillation frequencies in case of $\rho_{r}$ phase $=\pi / 2$ are slightly higher than those in case of $\rho_{r}$ phase $=-\pi / 2$ ([Fig. 1]).

\section{$3.4 \rho_{r}$ phase $=0$ and $\delta L>0$}

As shown in [Fig. 4], at a fixed $\kappa L$ value the largest gap in the oscillation gain between the first and second modes occurs at $\kappa L=10$. At the same $\kappa L$ value, the oscillation frequencies in case of $\rho_{r}$ phase $=0$ are slightly higher than those in case of $\rho_{r}$ phase $=-\pi / 2$ ([Fig. 1]). 


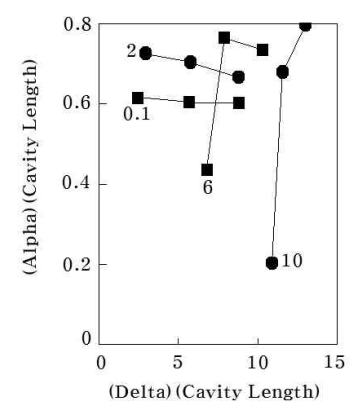

[Fig. 3] The graph of $(\alpha L, \delta L)$ in case of $\rho_{l}$ phase $=\pi, \rho_{r}$ phase $=\pi / 2$, and $\delta L>0$ as $\kappa L$ increases.

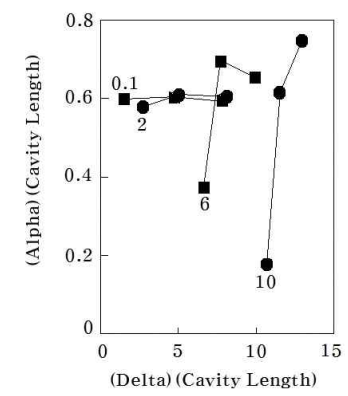

[Fig. 4] The graph of $(\alpha L, \delta L)$ in case of $\rho_{l}$ phase $=\pi, \rho_{r}$ phase $=0$, and $\delta L>0$ as $\kappa L$ increases.

So far, we have investigated the case of $\delta L>0$, but now we will examine the case of $\delta L<0$.

$3.5 \rho_{r}$ phase $=-\pi / 2$ and $\delta L<0$

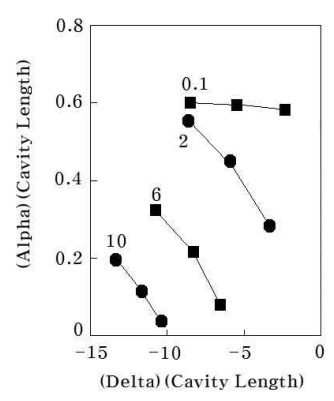

[Fig. 5] The graph of $(\alpha L, \delta L)$ in case of $\rho_{l}$ phase $=\pi, \rho_{r}$ phase $=-\pi / 2$, and $\delta L<0$ as $\kappa L$ increases. As shown in [Fig. 5], when $\kappa L$ increases from 0.1 to 10, the oscillation gain gradually 
decreases with increasing light feedback, similar to the case of $\rho_{r}$ phase $=-\pi / 2$ and $\delta L>0$ ([Fig. 1]). At the same $\kappa L$ value, the oscillation gains in case of $\rho_{r}$ phase $=\pi / 2$ and $\delta L<0$ are much lower than those in case of $\rho_{r}$ phase $=-\pi / 2$ and $\delta L>0$ ([Fig. 1]). At a fixed $\kappa L$ value, the largest gap in the oscillation gain between the first and second modes occurs at $\kappa L$ $=2$.

$3.6 \rho_{r}$ phase $=\pi$ and $\delta L<0$

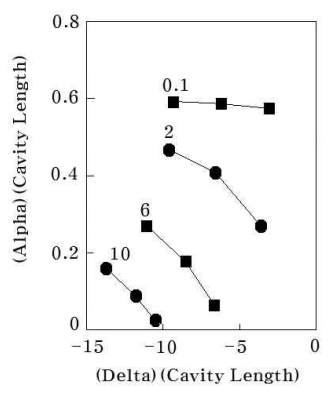

[Fig. 6] The graph of $(\alpha L, \delta L)$ in case of $\rho_{l}$ phase $=\pi, \rho_{r}$ phase $=\pi$, and $\delta L<0$ as $\kappa L$ increases.

As shown in [Fig. 6], at the same $\kappa L$ value, the oscillation gains in case of $\rho_{r}$ phase $=\pi$ are slightly lower than those in case of $\rho_{r}$ phase $=-\pi / 2$ ([Fig. 5]). At a fixed $\kappa L$ value, the largest gap in the oscillation gain between the first and second modes occurs at $\kappa L=2$.

$3.7 \rho_{r}$ phase $=\pi / 2$ and $\delta L<0$

As shown in [Fig. 7], at the same $\kappa L$ value, the oscillation gains in case of $\rho_{r}$ phase $=\pi / 2$ are slightly higher than those in case of $\rho_{r}$ phase $=-\pi / 2$ ([Fig. 5]). At a fixed $\kappa L$ value, the largest gap in the oscillation gain between the first and second modes occurs at $\kappa L=6$.

$3.8 \rho_{r}$ phase $=0$ and $\delta L<0$

As shown in [Fig. 8], at the same $\kappa L$ value, the oscillation gains in case of $\rho_{r}$ phase $=0$ are slightly higher than those in case of $\rho_{r}$ phase $=-\pi / 2$ ([Fig. 5]). At a fixed $\kappa L$ value, the largest gap in the oscillation gain between the first and second modes occurs at $\kappa L=10$. 


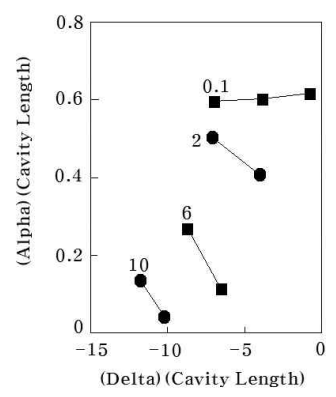

[Fig. 7] The graph of $(\alpha L, \delta L)$ in case of $\rho_{l}$ phase $=\pi, \rho_{r}$ phase $=\pi / 2$, and $\delta L<0$ as $\kappa L$ increases.

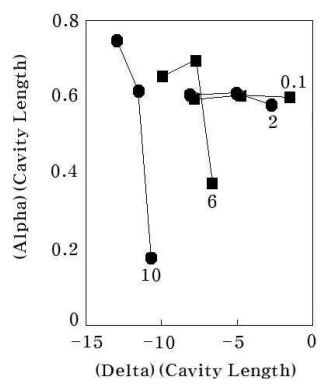

[Fig. 8] The graph of $(\alpha L, \delta L)$ in case of $\rho_{l}$ phase $=\pi, \rho_{r}$ phase $=0$, and $\delta L<0$ as $\kappa L$ increases.

\section{Conclusion}

While the grating phase of the left mirror face is fixed to be $\pi$, the oscillation frequency and threshold gain characteristics of DFB lasers are theoretically analyzed as the grating phase of the right mirror face is varied. The case was divided into Bragg deviation $\delta L>0$ and $\delta L<0$. In order to obtain single-mode operation, low frequency change, and stable frequency operation during high frequency current modulation, the optimum coupling strength $\kappa L$ values and grating phases of the right mirror face have been obtained.

It can be seen that when the grating phase of the right mirror surface is 0 , the degeneracy of the oscillation wavelength occurs in a DFB laser, which is not preferable. At a fixed $\kappa L$ value, the largest gap in the oscillation gain between the first and second modes occurs at $\kappa L$ $=10$.

In case of $\delta L>0$, DFB lasers should have $\kappa L=10$, in order to obtain the largest gap in the oscillation gain between the first and second modes. In case of $\delta L<0$, the oscillation frequency selectivity of the single-mode is excellent in the range from $\kappa L=2$ to $\kappa L=6$, except 
of $\rho_{r}$ phase $=0$. If the goal is to minimize the oscillation gain, DFB lasers should have $\kappa L=10$, in order to increase the feedback of the light.

\section{References}

[1] T.L. Koch, U. Koren, Semiconductor lasers for coherent optical fiber communications, Journal of Lightwave Technology, (1990), Vol.8, No.3, pp.274-293.

[2] H. Olesen, J. Salzman, B. Jonsson, B. Tromborg, Single-mode stability of DFB lasers with longitudinal Bragg detuning, IEEE Photonics Technology Letters, (1995), Vol.7, Issue.5, pp.461-463.

[3] S.K.B. Lo, H. Ghafouri-Shiraz, A method to determine the above-threshold stability of distributed feedback semiconductor laser diodes, Journal of Lightwave Technology, (1995), Vol.13, No.4, pp.563-568.

[4] C.A. Ferreira Fernandes, Stability in single longitudinal mode operation in DFB laser structures, The 12th IEEE Mediterranean Electrotechnical Conference, (2004), May 12-15; Dubrovnik, Croatia.

[5] Jing-Yi Wang, M. Cada, Jin Sun, Theory for optimum design and analysis of distributed-feedback lasers, IEEE Photonics Technology Letters, (1999), Vol.11, Issue,1, pp.24-26.

[6] M. Okai, S. Tsuji, N. Chinone, Stability of the longitudinal mode in lambda/4-shifted InGaAsP/InP DFB lasers, IEEE Journal of Quantum Electronics, (1989), Vol.25, Issue.6, pp.1314-1319.

[7] T. Yamanaka, S. Seki, K. Yokoyama, Numerical analysis of static wavelength shift for DFB lasers with longitudinal mode spatial hole burning, IEEE Photonics Technology Letters, (1991), Vol.3, Issue.7, pp.610-612.

[8] G. Morthier, R. Baets, Design of index-coupled DEB lasers with reduced longitudinal spatial hole burning, Journal of Lightwave Technology, (1991), Vol.9, No.10, pp.1305-1313.

[9] T.K. Sudoh, Y. Nakano, K. Tada, K. Kikuchi, T. Hirata, H. Hosomatsu, Self-suppression effect of longitudinal spatial hole burning in absorptive-grating gain-coupled DFB lasers, Photonics Technology Letters IEEE, (1993), Vol.5, No.11, pp.1276-1278.

[10] X. Pan, H. Olesen, B. Tromborg, Spectral linewidth of DFB lasers including the effects of spatial hole-burning and nonuniform current injection, Photonics Technology Letters IEEE, (1990), Vol.2, No.5, pp.312-315.

[11] Kee-Young Kwon, Jang-Geun Ki, Simulation and Examination for Beam Profile of DFB Laser, Journal of software assessment and valuation, (2019), Vol.15, No.1, pp.71-78. 